\title{
A violência como um problema de saúde e seu enfrentamento na Atenção Primária: uma revisão narrativa
}

\author{
Violence as a health problem and its confrontation in Primary Care: a narrative review \\ La violencia como problema de salud y su enfrentamiento en Atención Primaria: una \\ revisión narrativa
}

\begin{abstract}
David Ximenes de Araújo Neto ${ }^{1 *}$, Ariane Rodrigues da Silva ${ }^{1}$, Adriana de Cassia Silva Dias ${ }^{1}$, Júlia Cardoso Nunes ${ }^{1}$, Osmar Régis de Sousa Junior ${ }^{1}$, Pamella Cerqueira da Rocha de Lucena Oliveira $^{1}$, Raíza da Silva Pereira ${ }^{1}$, Yanne Fernanda de Barros Rola1.
\end{abstract}

\section{RESUMO}

Objetivo: Esclarecer as consequências da violência urbana na saúde de quem convive com tal fenômeno e identificar as estratégias de enfrentamento da violência pela atenção primária à saúde. Revisão bibliográfica: A vulnerabilidade está inerente a cada indivíduo que por sua vez possa vir a possuir um risco associado, variando de acordo com sua etnia, idade, classe social e sexualidade, entre outros. Com isso, são analisadas as implicações da violência na atenção primária de saúde, tanto para profissionais, para os serviços prestados, quanto para usuários das unidades básicas de saúde. Uma vez que a lógica de um território vivo pode alterar as percepções de violência, limitando ou expandindo as ações necessárias a serem tomadas no território adscrito. Considerações finais: É possível identificar e controlar casos de violência pela notificação dentro da atenção primária. Porém, isso não ocorre devido a uma baixa adesão da equipe profissional, o que aumenta os riscos e vulnerabilidades associados.

Palavras-chave: Violência, Saúde pública, Atenção primária à saúde.

\section{ABSTRACT}

Objective: Clarify the consequences of urban violence on health of those who live in such phenomenon and identify the consequences of coping with violence by primary health care. Bibliographic review: Vulnerability is inherent to each individual who in turn may have an associated risk, varying according to their ethnicity, age, social class and sexuality, among others. Thus, the implications of violence in primary health care are analyzed, both for professionals, for the services provided, and for users of basic health units. Since the logic of a living territory can alter perceptions of violence, limiting or expanding the necessary actions to be taken in the territory assigned. Final considerations: It is possible to identify and control cases of violence through notification within primary care. However, this is not due to the low adherence of the professional team, which increases the associated risks and vulnerabilities.

Keywords: Violence, Public health, Primary helth care.

\section{RESUMEN}

Objetivo: Esclarecer las consecuencias de la violencia urbana en la salud de quienes conviven con este fenómeno y identificar las consecuencias del afrontamiento de la violencia desde la atención primaria de salud. Revisión bibliográfica: La vulnerabilidad es inherente a cada individuo que a su vez puede tener un riesgo asociado, variando según su etnia, edad, clase social y sexualidad, entre otros. Así, se analizan las

${ }^{1}$ Universidade Estácio de Sá (UNESA), Rio de Janeiro - RJ. *E-mail: davidximenes13@gmail.com 
implicaciones de la violencia en la atención primaria de salud, tanto para los profesionales, para los servicios prestados, como para los usuarios de las unidades básicas de salud. Dado que la lógica de un territorio vivo puede alterar las percepciones de la violencia, limitando o ampliando las acciones necesarias a realizar en el territorio asignado. Consideraciones finales: Es posible identificar y controlar los casos de violencia a través de la notificación dentro de la atención primaria. Sin embargo, esto no se debe a la baja adherencia del equipo profesional, lo que aumenta los riesgos y vulnerabilidades asociados.

Palabras clave: Violencia, Salud pública, Atención primaria de salud.

\section{INTRODUÇÃO}

A violência é um fenômeno social amplo e complexo, que impacta fortemente na mortalidade da população, e tem significados diversos conforme o contexto. Decerto, essa mazela social pode ser definida como um comportamento de pessoas, grupos, classes ou povos, os quais geram estragos e prejuízos materiais e/ou psicológicos, por exemplo, agressão física, violência psicológica, violência institucional, entre outros, à vítima e também ao agressor (SANTOS MS, et al., 2017).

No que concerne a esse contexto, essa problemática afeta, de forma diferente, a população, levando a riscos diferenciados para os indivíduos, pois se deve levar em conta o gênero, a raça/cor, a idade e a sexualidade, tendo em vista as diversas vulnerabilidades socioeconômicas na sociedade hodierna, causadas pela carência de recursos como a falta de condições dignas de moradia, de saneamento básico, de energia, de água e de serviços de saúde, a qual atinge grande parte da população contribuindo para a exclusão social, intensificada pelo crescimento acelerado e desorganizado, o qual tornou essas vulnerabilidades sociais mais expressivas (BARBAR EM, 2018).

Dessa forma, nota-se que essas desigualdades comprometem o desenvolvimento, de forma saudável, dos indivíduos, uma vez que seus corpos, seus direitos e suas essências são constantemente violadas pelo Estado e pela sociedade, favorecendo, desse modo, o processo de adoecimento (SANTOS RSD, et al., 2020)

Nesse sentido, o sistema de saúde é um espaço onde a violência pode ser abordada, onde diversos profissionais podem contribuir para a prevenção, atenção e desenvolvimento de ações que possam colaborar para o enfrentamento do problema, sendo a Atenção Básica (AB) a principal porta de entrada e de comunicação com as Redes de Atenção à Saúde (RAS) do Sistema Único de Saúde (SUS). Sendo essa responsável por coordenar os cuidados, fluxos e contrafluxos de pessoas nas RAS, de forma integral, referente às suas necessidades e demandas da localidade, analisando também os fatores determinantes e condicionantes da saúde (RODRIGUES EAS, et al., 2018).

Outrossim, é fulcral ressaltar a importância da Atenção Primária à Saúde (APS) como estratégia de intervenção e de enfrentamento à violência como um problema de saúde pública, uma vez que a APS pode contar com a Estratégia de Saúde da Família (ESF), a qual é pautada no cuidado centrado na pessoa e na família, na integralidade do indivíduo, na coordenação do cuidado, na participação popular e na atuação intersetorial. Em face disso, percebe-se que o uso desses recursos é imprescindível no diagnóstico do problema, na mitigação da causa (prevenção de doenças) e na promoção da saúde (PERES AM, et al., 2020).

Ainda sob a óptica supracitada, a violência quando não é fatal, pode causar distúrbios de ansiedade e alimentares, depressão, alteração de humor, como também pode levar ao uso de drogas lícitas e ilícitas. Tal realidade acarreta a sobrecarga no serviço de saúde (ROCHA GVL, et al.,2020) Dessarte, esse cenário caótico urge de uma intervenção pautada em uma abordagem ampla, integral e multidisciplinar, com o fito de atenuar e de prevenir os impactos negativos causados (NONATO LOF, et al., 2020).

Frente ao contexto exposto, o objetivo desse estudo foi esclarecer as consequências da violência urbana na saúde de quem convive com tal fenômeno e identificar as estratégias de enfrentamento da violência pela atenção primária à saúde. 


\section{REVISÃO BIBLIOGRÁFICA}

\section{A Violência como um Fenômeno Social Complexo}

O Sistema Único de Saúde é responsável pelas condições de saúde e pelo serviço prestado à população e, com isso, busca reafirmar o dever do Estado em proporcioná-los a sociedade. A Organização Mundial da Saúde (OMS), por meio da Assembleia Geral das Organizações das Nações Unidas (ONU), considerou em 1983 a violência como um importante desafio para a saúde pública e, assim, é fundamental que seja feita a notificação de casos de suspeita ou de confirmação (EGRY EY, et al., 2018).

É importante notificar todo tipo de violência, por exemplo, doméstica, infantil, contra homossexuais, travestis, transsexuais. Dentre essas violências, as físicas e psicológicas. A atenção primária de saúde é, sem dúvidas, muito importante nesse processo, pois é um dos primeiros serviços a serem buscados já que são porta de entrada para o sistema de saúde. Deve-se considerar que a APS trabalha na articulação de assistência curativa, com prevenção e promoção da saúde e, deve ser procurada mesmo na ausência de queixas ligadas a adoecimentos já manifestos (D'OLIVEIRA AFPL, et al., 2020).

Uma forma de controlar os casos de violência é informá-los à instituição de saúde da região. Isso é feito através dos agravos de notificação compulsória e é uma ação completamente obrigatória para os profissionais de saúde e/ou para qualquer pessoa que seja responsável pelo local. É realizado em qualquer instituição, pública e privada, sempre que há suspeita de casos ou quando já é confirmado. Todavia, o que acontece com frequência na prática é o descaso quanto a essa denúncia, o que torna difícil a resolução do problema pois diversos casos permanecem sem serem identificados (EGRY EY, et al., 2018; SCHEK G, et al., 2017).

É essencial destacar, também, como esse trabalho de combate à violência deve ser feito por uma equipe multidisciplinary. No entanto, não é assim que acontece na prática. O desenvolvimento desse serviço acaba sendo feito de forma individual e sem conexão com toda a equipe, o que torna o resultado ineficaz. Por isso, é muito importante que haja o envolvimento de todas as áreas de saúde, com ações multidisciplinares, como o atendimento e acolhimento para depois encaminhar para as redes de cuidados e proteção social. Com isso, deve-se atentar à integralidade do cuidado, ao vínculo e ao trabalho de forma multi e interdisciplinar (SCHEK G, et al., 2017; NONATO LOF, et al., 2020).

\section{Vulnerabilidade social e violência}

Segundo Nonato LOF, et al. (2020), a APS territorializada se dá a partir de uma determinada população adscrita em um local delimitado; este território é muito dinâmico e devido a isso as vulnerabilidades e constantes riscos ocorrem com frequência.

Este território, por ser dinâmico e com grandes recorrências de riscos e mudanças, se caracteriza como uma reprodução de processo saúde-doença epidemiologicamente relevante, sendo assim, um processo que engloba fatores politicos e sociais, influenciando no modo em que as pessoas vivem e sobrevivem, além de toda a equipe que ali também se expõe e norteia o reconhecimento dos fatores determinantes de saúde até o planejamento e execução de suas ações a serem desenvolvidas e colocadas em prática.

O enfrentamento da violência para todos que ali estão sejam profissionais ou moradores, é um problema recorrente que causa muita tensão, medo, frustação, impotência e angústia. E mesmo diante de todo cenário os profissionais ali envolvidos não deixam de ofertar um cuidado integral, manter um vínculo com toda a população, transcendendo as limitações que o território apresenta (NONATO LOF, et al., 2020; CARDOSO MCC, et al., 2016; PINTO AGA, et al., 2017).

Por mais que a violência esteja presente e seja vivenciada na grande maioria dos territórios em que a Atenção Primária a Saúde esteja atuando, o seu enfrentamento por toda equipe multidisciplinar é de suma importância para a elaboração de estratégias de prevenção contra este problema. É esperado que todo enfrentamento, diante de políticas públicas e estratégias de gerenciamento, estabelecendo vinculo e organização do trabalho, seja feito de forma a atender às necessidades que o território e a população necessita para ter dignidade no processo de saúde e também que o cenário exposto tendo em vista violência como um problema bem citado que englobe saúde pública seja fator visto, gerenciado, pois ainda há pouca visibilidade entre os profissionais e gestores de saúde (NONATO LOF, et al., 2020). 


\section{A Violência Como um Problema de Saúde}

A violência se configura como um problema social com forte impacto na saúde e vem sendo reconhecida na área da saúde como demanda para a oferta assistencial e para a atenção integral da população. Esse fenômeno complexo e multideterminado histórica, social e culturalmente, interfere diretamente na deterioração das condições de saúde e qualidade de vida da sociedade, e dos gastos públicos. Suas consequências se expressam em diferentes âmbitos da vida e das relações humanas e sociais, inclusive no processo saúde-adoecimento (SANTOS MS, et al., 2017; MENDONÇA CS et al., 2020).

Ao se analisar que a violência, por provocar diversas consequências multifatoriais (letalidade, conflitos interpessoais, interterritoriais, assaltos, agressões, entre outros), não só a um grupo de pessoas, ou a um único individuo, mas à população toda, e no território em que está inserido, foi incluído na agenda do setor por meio da Política Nacional de Redução da Mortalidade por Acidentes e Violência em 2001, o seu enfretamento, na tentativa de que seu este seja melhor aplicado e desenvolvido quando se tem atenção dos serviços de saúde (SANTOS MS, et al., 2017; MOURA LBA, et al., 2015; RIBEIRO WS, et al., 2013).

Entretanto, os trabalhos das equipes de estratégias de saúde nestes territórios têm sido limitados, devido aglomerações, situações precárias e desigualdade social; tornando o trabalho destes profissionais nestas áreas inseguro, restringindo assim a atenção em saúde somente aos usuários que frequentam as unidades (POLARO SHI, et al., 2013).

Existe a necessidade de apoio das autoridades públicas para que se possam desenvolver estratégias que auxiliem e facilitem a assistência tanto aos usuários da unidade que estão inseridos no território, quanto aos profissionais que desempenham a assistência junto a estas areas vulneráveis; sendo assim, espera-se que o desenvolvimento de estratégias de cuidado que amplie a assistência à saúde possa ser urgentemente colocado em prática para a população, e o convívio necessário para desenvolver o trabalho de forma eficaz não seja arruinado pelas adversidades que ali são enfrentadas (SANTOS MS, et al., 2017).

\section{Os Desafios da Atenção Primária Frente a Territórios Violentos}

Nonato LOF, et al. (2020), demonstra em sua pesquisa que uma das consequências do atendimento em territórios violentos é o impacto nos profissionais, causando-lhes sentimentos prejudiciais à sua saúde mental. Além disso, nas ações em territórios violentos, ainda há riscos que não há como evitar. Assim, a proteção dos trabalhadores de saúde enquanto prestadores imparciais de cuidado é, por diversas vezes, desrespeitada pelos atores envolvidos nos serviços, sendo estes estatais ou não (BARBAR AEM, 2018).

A violência causa impacto diretamente aos profissionais e, por consequência, na qualidade dos serviços ofertados à população, muitas vezes diminuindo o acesso desta aos serviços ou até prejudicando a realização dos serviços pelos profissionais de saúde na comunidade (NONATO LOF, et al., 2020). O cuidado em saúde em áreas violentas demanda a adequação das atividades à realidade local, podendo representar inúmeros desafios para sua promoção efetiva. Ademais, a insegurança, infraestrutura inadequada, convívio com o tráfico de drogas, entre outros fatores, trazem inúmeras limitações para o acesso à saúde, tornando árduas as práticas de promoção e prevenção, mais uma vez limitando o acesso da comunidade à saúde (SANTOS MS, et al., 2017).

Diante de evento violento no território, urge a necessidade de replanejamento das atividades, que acabam sendo reagendadas. Quando a unidade mantém o seu funcionamento em dia de maior violência, o número de faltas às consultas aumenta significativamente e a demanda por serviços de saúde fica reduzida, limitando a prática profissional (MACHADO CB, et al., 2015). Essa premissa é evidenciada por Polaro SHI, et al. (2013) demonstrando, em sua experiência com profissionais de áreas violentas, que a violência faz com que o trabalho na ESF seja limitado, pois ao restringir-se a atender no interior das unidades de saúde, o atendimento à comunidade fica prejudicado. Nesse sentido, a violência é geradora de lacunas no processo de cuidar (MACHADO CB, et al., 2015)

O último e, de certa forma, o maior desafio a ser debatido para o trabalho do profissional de saúde perante à violência armada é responder às demandas subjetivas de cuidado que surgem da comunidade e dos usuários da APS, visto que a grande maioria das consequências da violência se manifestam de forma 
mais tênue. São queixas como pressão arterial aumentada ou crises de pânico, por exemplo. Também há as vítimas secundárias, que não sofreram diretamente no momento, mas que foram afetadas de alguma forma, como familiares de pessoas assassinadas ou desaparecidas ou usuários que presenciaram cenas de violência; podendo da mesma forma gerar traumas. Assim, é essencial compreender amplamente o contexto, o monitoramento compartilhado de eventos adversos (como mortes, sequestros, tiroteios), realizar a escuta cuidadosa, empática e atenta na clínica e o trabalho multidisciplinar, com o fito de ajudar a responder de forma mais efetiva, aliando-se à resposta puramente biomédica (BARBAR AEM, 2018)

\section{Estratégias de Enfrentamento à Violência em Unidades de Atenção Primária}

A abordagem das situações de violência não é simples, sequer há soluções prontas. Na realidade, este é um dos maiores desafios do SUS e, certamente, um de seus limites. A APS, enquanto promotora da aproximação com os usuários, tem o poder de estreitar o vínculo, estabelecer relações de confiança e favorecer a criação de espaços de diálogo e participação com a comunidade, sendo, então, uma forte possibilidade de enfrentamento desta problemática. Nesse sentido, é de extrema importância que a voz do usuário seja protagonista na identificação de demandas e entendimento da realidade da comunidade dentro da qual a clínica vai se desenvolver (MACHADO CB, et al., 2015; RODRIGUES EAS, et al., 2018).

Dessa forma, fica claro que o engajamento da comunidade é primordial para que o cotidiano de violência seja abertamente abordado e enfrentado, integrado ao cuidado em saúde, e para que as intervenções da APS nestes territórios possam ser transformadoras daquela realidade (MACHADO CB, et al., 2015; RODRIGUES EAS, et al., 2018).

As reuniões de equipe entre os profissionais da ESF são importantes para ajudar a definir estratégias gerais de ações com a comunidade, possibilitando grande aprendizado por meio das vivências entre os profissionais, diminuindo angústias pessoais dos membros da equipe de saúde e ainda contribuindo para resolver os casos com a colaboração do grupo a partir de suas diferentes visões e conhecimentos (KANNO NP, et al., 2012; (MACHADO CB, et al., 2015).

Para Nonato LOF (2018), a comunicação é a estratégia central com relação à violência no território, tendo o ACS e as lideranças comunitárias como informantes fundamentais nesse processo. Os ACS são, nesse contexto, profissionais estratégicos no contato com a equipe acerca de possíveis situações de violência no território, pois fortalecem e estreitam o vínculo entre o serviço e o usuário, além de possuírem a particularidade de pertencer àquela comunidade, sendo um profissional motivador ao protagonismo social e ao cuidado à saúde no território.

Ainda, observa-se que a comunicação é fundamental para a tomada de decisão e consequentemente uma aliada para o gerenciamento e organização do processo e trabalho, em serviços expostos à violência urbana. Em geral, estudos descrevem as estratégias de comunicação utilizadas para avisar uns aos outros quando há perigo ou risco no território, entre elas um aplicativo de celular. Como meio de proteção, também é pautado o uso do jaleco dentro e fora da unidade básica de saúde, demonstrando a preocupação dos mesmos em serem reconhecidos como profissionais de saúde no território. É uma maneira de identificação à distância e por eventuais pessoas que não os conheçam na rua. Cabe ao gerente da unidade o reconhecimento do risco levantado pela equipe e o incentivo ao uso do jaleco por profissionais, estagiários, residentes dentre outros trabalhadores que possam ofertar serviços de saúde à população (NONATO LOF, 2018)

Na literatura, pode-se encontrar o uso do jaleco dentro e for a da unidade básica com duas finalidades: identificar o profissional de saúde e servir como equipamento de proteção individual (EPI). Relacionado à biossegurança está veiculado à Lei Federal no 11.105, de 25 de março de 2005 (BRASIL, 2005) e quanto à utilização institucional como forma de identificação pessoal, atua para proteção do funcionário, reforçando a tese de Fontanella BJB (2012) com relação à identificação, ao afirmar quando o profissional de saúde porta um jaleco ou apresenta-se de branco, em um ambiente assistencial, a ideia é apenas dizer que o indivíduo se comporta como alguém institucionalmente reconhecido, como um cartão ou crachá de identidade (NONATO LOF, et al., 2020). 


\section{CONSIDERAÇÕES FINAIS}

Este trabalho pode contribuir como forma de nortear olhar para a questão da violência e sua abordagem dentro do SUS. O sistema de saúde brasileiro é um espaço onde a violência pode e deve ser debatido. Diversos profissionais podem contribuir para a prevenção, atenção e desenvolvimento de ações que possam colaborar para o enfrentamento do problema, sendo a Atenção Básica a principal porta de entrada e de comunicação. Os estudos realizados e apresentados nessa revisão, sinalizam que na prática diária os casos permanecem sem notificação devido à falta de participação do profissional em denunciar a violência. $\mathrm{Na}$ presente exposição, entendemos como a Saúde, como instituição, consegue identificar e controlar os casos através dos agravos de notificação compulsória, realizada por profissionais de área através de comunicado a autoridade local.

\section{REFERÊNCIAS}

1. BARBAR AEM. Atenção primária à saúde e territórios latino-americanos marcados pela violência. Revista Panamericana de Salud Pública, 2018; 42: e142

2. BRASIL. Lei no 11.105, de 25 de março de 2005. Disponível em: http://www.planalto.gov.br/ccivil_03/_ato20042006/2005/lei/l11105.htm. Acessado em: 30 de março de 2021.

3. CARDOSO MCC, et al. Moral Distress in Family Health Strategy: experiences expressed by daily life. Ver Esc Enferm USP, 2016;50(n.spe):89-95.

4. D'OLIVEIRA AFPL, et al. Obstáculos e facilitadores para o cuidado de mulheres em situação de violência doméstica na atenção primária em saúde: uma revisão sistemática. Interface-Comunicação, Saúde, Educação, $2020 ; 24$.

5. EGRY EY, et al. Notificação da violência infantil, fluxos de atenção e processo de trabalho dos profissionais da Atenção Primária em Saúde. Ciência \& Saúde Coletiva, 2018; 23: 83-92.

6. FONTANELLA BJB, et al. Rituais e símbolos na atenção formal à saúde: o caso do vestuário profissional na ótica de pacientes da Atenção Básica. Physis Revista de Saúde Coletiva, 2012; 22: 507-52

7. GONÇALVES HCB, et al. Violência urbana e saúde mental: desafios de uma nova agenda? Fractal: Revista de Psicologia, 2017; 29: 17-23.

8. KANNO NP, et al. Profissionais da Estratégia Saúde da Família diante de demandas médico-sociais: dificuldades e estratégias de enfrentamento. Saúde e sociedade, 2012; 21(4): 884-894

9. MACHADO CB, et al. Violência urbana: repercussões e consequências na assistência à saúde em uma Unidade de Saúde da Família. Ciência, Cuidado e Saúde, 2015; 14(4): 1445-1452.

10. MENDONÇA CS, et al. Violência na Atenção Primária em Saúde no Brasil: uma revisão integrativa da literatura. Ciência e Saúde Coletiva, 2020; 25: 2247-2257.

11. MOURA LBA, et al. Violência e juventude em um território da Área Metropolitana de Brasília, Brasil: uma abordagem socioespacial. Ciência Saúde Coletiva, 2015; 20(11):3395-405.

12. NONATO LOF. Gerenciamento De Serviços De Atenção Primária À Saúde Em Territórios Expostos À Violência Urbana. Dissertação (Mestrado em Enfermagem) - Universidade Federal do Paraná, Curitiba, 2018; 113p

13. NONATO LOF, et al. Estratégias de gerenciamento na Atenção Primária à Saúde em territórios de vulnerabilidade social expostos à violência. Revista da Escola de Enfermagem da USP, 2020; 54.

14. PINTO AGA, et al. Experiences in the Family Health Strategy: demands and vulnerabilities in the territory. Rev Bras Enferm. 2017; 70: 920-927.

15. POLARO SHI, et al. Enfermeiras desafiando a violência no âmbito de atuação da Estratégia de Saúde da Família. Texto \& Contexto-Enfermagem, 2013; 22(4): 935-942.

16. RIBEIRO WS, et al. The impact of epidemic violence on the prevalence of psychiatric disorders in São Paulo and Rio de Janeiro, Brazil. PLoS One, 2013; 8.

17. ROCHA G VL, et al. Representações sociais de pessoas usuárias da Atenção Primária à Saúde sobre violência: estudo de gênero. Rev Gaúcha Enferm. 2020; 41: e20190286.

18. RODRIGUES EAS, et al. Violência e atenção primária à saúde: percepções e vivências de profissionais e usuários. Saúde em Debate, 2018; 42: 55-66.

19. SANTOS MS, et al. O enfrentamento à violência no âmbito da estratégia saúde da família: desafios para a atenção em saúde. Revista Brasileira em Promoção da Saúde, 2017; 30(2): 229-238.

20. SANTOS RSD, et al. Armed conflict at primary care: challenges for practice and training. Revista Brasileira de Enfermagem, 2020; 73(2).

21. SCHEK, Gabriele et al. Organização das práticas profissionais frente à violência intrafamiliar contra crianças e adolescentes no contexto institucional. Revista Latino-Americana de Enfermagem, 2017; 25 